\title{
Performances de croissance et composition corporelle de taureaux Charolais en stations de contrôle individuel
}

\author{
G Renand 1, Y Geay 2, F Ménissier 1 \\ avec la collaboration technique de $\mathrm{C}$ Barboiron ${ }^{2}$ et $\mathrm{P}$ Gillard 1 \\ 1 Station de génétique quantitative et appliquée, Centre de recherche Inra de Jouy-en-Josas, \\ 78352 Jouy-en-Josas cedex; \\ 2 Laboratoire croissance et métabolisme des herbivores, \\ Centre de recherche Inra de Theix, 63122 Saint-Genès-Champanelle, France
}

(Reçu le 1er février 1995 ; accepté le 7 avril 1995)

\begin{abstract}
Résumé - Dans deux stations de contrôle individuel, 510 taureaux Charolais ont été contrôlés sur leur croissance ( $P F$ : poids final) et leur efficacité alimentaire ( $C R$ : consommation résiduelle, écart entre consommation observée et consommation théorique compte tenu du poids et du gain de poids). Deux échantillons de 70 taureaux présentant des valeurs extrêmes pour un indice $\left(l_{S}\right)$ combinant les deux caractères précédents ont été retenus. Leur composition corporelle a été estimée par la technique de diffusion de l'eau lourde, simultanément à la mesure de la taille des adipocytes. Ces mesures ont permis de montrer qu'il existe une importante variabilité de la teneur en dépôts adipeux entre individus, indépendante de leur poids (PF) et légèrement corrélée avec le critère d'efficacité (CR). La différence créée entre les deux échantillons ( $+96 \mathrm{~kg}$ de poids final et $-1,7 \mathrm{~kg} / \mathrm{jou}$ de consommation résiduelle) va de pair avec une teneur en dépóts adipeux totaux sensiblement plus faible $(-8 \mathrm{~g} / \mathrm{kg}$ de poids vif) chez les taureaux ayant des indices $\left(l_{S}\right)$ supérieurs par rapport aux taureaux ayant des indices inférieurs. Ces résultats montrent la possibilité de sélectionner indirectement les taureaux sur leur croissance musculaire à partir de la mesure de leur croît et de leur efficacité alimentaire. Toutefois, la corrélation élevée entre le diamètre des adipocytes et la teneur en dépôts adipeux $(r=+0,56)$ indique que cette sélection peut être améliorée en intégrant la mesure de la taille des adipocytes.
\end{abstract}

bovin à viande / contrôle individuel / composition corporelle / eau lourde / adipocytes

Summary - Growth performances and body composition of Charolais bulls in central test stations. In two central test stations, 510 Charolais bulls were tested for their growth (PF = final weight) and feed efficiency (CR = residual feed intake, difference between the actual intake and the predicted intake adjusted for weight and weight gain). Two groups of 70 bulls each were chosen among 
extreme values of a selection index $\left(\mathrm{I}_{\mathrm{S}}\right)$ using both traits. Their body composition was estimated by the deuterium oxide diffusion technique and by measuring subcutaneous adipose cell size. These measurements show that fatness varies greatly among animals and is independent of their weight (PF) and slightly dependent on their feed efficiency (CR). The two groups of bulls studied had large differences in body weight ( $+96 \mathrm{~kg}$ of final weight) and feed efficiency $(-1.7 \mathrm{~kg} /$ day of residual feed intake) together with significant differences in fatness $(-8 \mathrm{~g} / \mathrm{kg}$ of fat content). These results favoured bulls with the highest indexes $\left(\mathrm{I}_{\mathrm{S}}\right)$ and show that the muscle growth capacity of young bulls can be selected for using measurements of live growth rate and feed efficiency. The highly positive correlation between adipose cell diameter and fat content $(r=+0.56)$ indicates that this characteristic could also be used to further improve selection.

\section{beef cattle / central test station / body composition / deuterium / adipose cell}

\section{INTRODUCTION}

Dans les programmes d'amélioration génétique des races bovines destinées à la production de viande, le contrôle individuel des jeunes taureaux est une étape essentielle pour améliorer les aptitudes bouchères. En effet, la variabilité d'origine génétique de ces dernières est suffisamment élevée pour qu'une sélection de ces jeunes taureaux sur leurs propres performances permette d'améliorer les aptitudes des descendants (Renand et al, 1992).

Dans les troupeaux allaitants, les veaux mâles étant généralement commercialisés au sevrage, seules les performances antérieures à ce stade peuvent être effectivement contrôlées. II est donc intéressant de regrouper dans des stations, dès leur sevrage, les jeunes taureaux candidats à la reproduction. Cela permet de contrôler leurs propres performances sur des critères beaucoup plus proches des aptitudes recherchées chez les animaux de boucherie. Parmi ces critères, la croissance pondérale (gain moyen quotidien ou poids à âge type) ou la morphologie (mensurations ou notes de pointage) sont les plus faciles à mesurer. Le contrôle en station permet également la mesure des quantités d'aliments ingérés afin de mettre en évidence les différences d'efficacité alimentaire entre animaux. Toutefois, cette mesure n'est que rarement réalisée car elle nécessite des installations coûteuses. Enfin, s'il n'est évidemment pas possible de mesurer par abattage la composition corporelle de ces candidats à la sélection, le contrôle en station permet d'envisager une mesure indirecte de celle-ci à l'aide d'une estimation in vivo.

C'est ainsi qu'en France, et depuis de nombreuses années, les aptitudes bouchères des taureaux destinés à l'insémination artificielle sont contrôlées en station avant que ces animaux soient évalués sur descendance. Depuis le début des années 1980 , un nouveau protocole a été mis en place afin de favoriser la sélection des taureaux sur leur capacité de croissance musculaire puisque cette dernière apparaît comme l'objectif primordial pour améliorer l'efficacité de l'engraissement et la qualité des carcasses (Geay et Robelin, 1979 ; Ménissier et al, 1986). Conformément aux recommandations du groupe de travail de la Fédération européenne de zootechnie (Andersen et al, 1981), les jeunes taureaux reçoivent un régime alimentaire relativement peu encombrant et de niveau élevé afin de mettre en évidence les différences de croissance musculaire entre animaux (Ménissier, 1988). En l'absence d'outil de mesure in vivo de la composition corporelle, la croissance musculaire est indirectement sélectionnée à travers un indice qui combine la croissance et l'efficacité alimentaire. En effet, Geay et al (1971) ont montré qu'au 
sein d'une même race les animaux ayant le potentiel de croissance le plus élevé ont un coût énergétique du gain de poids vif le plus faible, après déduction des besoins d'entretien. L'efficacité alimentaire est ainsi en relation positive avec la proportion de muscles dans le gain de poids, puisque, pendant cette phase de croissance des jeunes, le coût énergétique d'accroissement de la masse maigre est inférieur à celui des dépôts adipeux (Geay et Robelin, 1979 ; Brelin et Brännang, 1982 ; Webster, 1985).

Toutefois ces relations entre individus, qui relient la vitesse de croissance, l'efficacité alimentaire et la composition corporelle, sont opposées aux relations induites par le niveau alimentaire, car l'accroissement de celui-ci se traduit par une augmentation des dépôts adipeux et une réduction de l'efficacité alimentaire (Geay et al, 1976 ; Robelin et Daenicke, 1980). En outre l'efficacité alimentaire d'un individu dépend d'autres facteurs que la composition corporelle, notamment les besoins d'entretien (Webster, 1989).

Il importait donc de vérifier si la sélection des taureaux sur leur vitesse de croissance et leur efficacité alimentaire permet de sélectionner les animaux ayant une croissance musculaire supérieure. Dans ce but, une expérimentation a été réalisée pour estimer les relations entre l'indice de sélection actuellement utilisé et la composition corporelle. Cette dernière a été estimée par une technique de référence basée sur la mesure de l'espace de diffusion de l'eau lourde (Robelin, 1982). Cette même expérimentation a également été conçue pour étudier l'intérêt de compléter le protocole actuellement appliqué par une estimation systématique de la composition corporelle en validant une nouvelle technique basée sur la mesure de la taille des adipocytes (Robelin et Agabriel, 1986). Le dispositif expérimental a été conçu pour obtenir des estimations précises des corrélations en ne contrôlant qu'un échantillon réduit d'animaux.

\section{MATÉRIEL ET MÉTHODES}

Pour cette expérimentation, 510 jeunes taureaux Charolais de deux centres de production de semence (UCATRC et OGER) furent utilisés. Ils furent contrôlés entre 1986 et 1990 dans deux stations : à Creuzier-le-Neuf (Allier) pour l'UCATRC et à Château-Gontier (Mayenne) pour I'OGER. Chaque année et dans chaque station, 46 à 69 jeunes taureaux furent contrôlés après avoir été répartis en deux groupes de contemporains en fonction de leur date de naissance. $\dot{A}$ l'intérieur d'un groupe, les différences d'âge sont inférieures à 6 semaines.

\section{Protocole national de contrôle individuel en station}

Dès leur entrée, effectuée à l'âge moyen de 10 mois, tous les animaux subissent une période d'adaptation d'au moins 8 semaines au régime alimentaire et au mode de stabulation. Ils sont ensuite contrôlés pendant 18 semaines. Ils reçoivent un aliment complet condensé $(57 \%$ de luzerne et $37 \%$ de maîs) distribué à volonté pendant les 4 premières semaines et restreint en fonction de leur poids pendant les 14 semaines suivantes. La quantité d'énergie (UFV) apportée à un taurillon de poids vif donné (PV) afin qu'il réalise le gain moyen quotidien (G) est la suivante: $\mathrm{UFV}=\alpha \mathrm{PV}^{0,75}+\beta \mathrm{PV}^{0,4} \mathrm{G}^{1,4}$ (Geay et Micol, 1988). Dans le cas des stations pour la race Charolaise, les quantités distribuées sont calculées avec une valeur de $\mathrm{G}=1,500 \mathrm{~kg} / \mathrm{jour}$.

A la fin des contrôles, un poids à âge type final (PF) est calculé ainsi qu'un critère d'efficacité alimentaire : la consommation résiduelle (CR). Cette dernière est calculée comme la différence entre la quantité d'énergie consommée (UFV) pendant les 14 dernières semaines de contrôle et la quantité qu'un taurillon de même poids vif moyen (PV) doit théoriquement consommer pour réaliser le même gain moyen quotidien $(G)$ : $\mathrm{CR}=\mathrm{UFV}-\left(\alpha \mathrm{PV}^{0,75}+\beta \mathrm{PV}^{0,4} \mathrm{G}^{1,4}\right)$. Chaque taureau est également jugé par un expert sur son développement musculaire (DM) et son développement squelettique (DS).

Pour chacun de ces caractères, un indice de valeur génétique $\left(I_{k}\right)$ est calculé selon la formule classique : $I_{k}=100+20 h_{k}^{2} \Delta_{k} / \sigma_{k}$ où $\Delta_{k}$ représente l'écart aux contemporains et $\sigma_{k}$ l'écart type phé- 
notypique du caractère. Dans cette formule, les coefficients d'héritabilité sont de $h^{2}=0,40$ pour le poids final et les notes de développement musculaire et squelettique et de $\mathrm{h}^{2}=0,35$ pour la consommation résiduelle (Renand et al, 1992). Enfin, en considérant que le poids final et la consommation résiduelle sont liés par une corrélation génétique de $r_{\mathrm{g}}=-0,55$, les taureaux ont été classés sur un indice de sélection $\left(I_{S}\right)$ qui combine les deux indices sur ces deux caractères $\left(I_{P F}\right.$ et $\left.I_{C R}\right)$ avec pour objectif l'obtention d'un progrès génétique relatif (en unité d'écart type du caractère) identique sur chacun d'eux : $I_{S}=122,9+0,426 I_{P F}-0,655 I_{C R}$.

\section{Échantillonnage des animaux expérimentaux}

Parmi ces 510 taureaux, 140 furent choisis sur la valeur de leur indice synthétique $\left(I_{S}\right): 70$ d'entre eux parmi les taureaux ayant les indices les plus faibles et 70 autres parmi les taureaux ayant les indices les plus forts. Ces 510 taureaux ayant été contrôlés dans deux stations pendant 5 années, le choix des $2 \times 70$ animaux expérimentaux fut réalisé en retenant sept taureaux extrêmes dans chaque station et chaque année.

\section{Mesure de la composition corporelle}

Le dernier jour des contrôles, la composition corporelle de ces 140 taureaux fut estimée par la méthode de diffusion de "l'eau lourde» qui est potentiellement la méthode la plus précise (Robelin, 1982). Cette technique repose sur l'existence d'une très étroite relation entre la teneur en lipides et la teneur en eau chez les jeunes bovins en croissance. Elle est basée sur l'estimation du volume hydrique d'un animal par mesure de l'espace de diffusion d'un marqueur, l'eau lourde $\left(\mathrm{D}_{2} \mathrm{O}\right)$ en l'occurrence. Une quantité connue de marqueur fut injectée par la veine jugulaire à raison de $0,5 \mathrm{~g} / \mathrm{kg}$ de poids vif. Des échantillons sanguins furent prélevés $6,8,24$ et 48 heures après l'injection en vue du dosage de l'isotope. L'espace de diffusion (ED) fut calculé en divisant la quantité de deutérium injectée par la concentration «initiale». Cette dernière, calculée pour chaque taureau, correspond à l'origine de la régression des concentrations sur le temps. Les taureaux ayant été pesés au moment de l'injection puis 24 et 48 heures plus tard, le poids des dépôts adipeux totaux (DA) fut estimé par l'équation : $\mathrm{DA}=0,690 \mathrm{PM}-0,838 \mathrm{ED}$ oủ $\mathrm{PM}$ représente la moyenne des trois pesées. L'écart type résiduel de cette équation de prédiction est de $5,0 \mathrm{~kg}$ (Robelin, 1982). La teneur en dépôts adipeux totaux (\%DA) fut calculée en divisant la masse estimée (DA) par le poids vif moyen (PM).

Le lendemain de l'injection, un échantillon d'environ $1 \mathrm{~g}$ de tissu adipeux sous-cutané fut prélevé par biopsie sur le haut de la cuisse puis plongé dans une solution d'acide osmique pour fixer les adipocytes, conformément au protocole proposé par Robelin et Agabriel (1986). Après quelques jours, l'échantillon fut dilacéré afin de dissocier les cellules adipeuses et de les photographier sous le microscope. Le diamètre moyen (Diam) d'une centaine de cellules fut mesuré sur une table à digitaliser.

\section{Estimation des corrélations dans la population totale}

Le processus d'échantillonnage retenu modifie les variances et covariances entre variables observées dans l'échantillon sélectionné par rapport à celles qui existent dans la population totale. Soit V la matrice des variances et covariances entre les variables dans la population totale non sélectionnée et $\mathbf{V}^{*}$ celle dans la population sélectionnée lorsque la sélection a porté sur la ou les variables représentées dans $\mathbf{V}_{11}$. Les variances et covariances de $\mathbf{V}^{\star}$ s'expriment en fonction de celles de V (Pearson, 1903):

$$
\begin{aligned}
& \text { Soit } \mathbf{V}=\left[\begin{array}{c}
\mathbf{V}_{11} \mathbf{V}_{12} \\
\operatorname{sym} \mathbf{V}_{22}
\end{array}\right] \\
& \text { et } \mathbf{V}^{*}=\left[\begin{array}{l}
\mathbf{v}_{11}{ }^{*} \mathbf{v}_{12}{ }^{{ }^{*}} \\
\operatorname{sym} \mathbf{v}_{22}{ }^{*}
\end{array}\right] \\
& =\left[\begin{array}{lc}
\mathbf{V}_{11}{ }^{*} & \mathbf{V}_{11}{ }^{*} \mathbf{V}_{11}^{-1} \mathbf{V}_{12} \\
\operatorname{sym} \mathbf{V}_{22}-\mathbf{V}_{21} \mathbf{V}_{11}^{-1}\left(\mathbf{V}_{11}-\mathbf{V}_{11}{ }^{*}\right) \mathbf{V}_{11}^{-1} \mathbf{V}_{12}
\end{array}\right]
\end{aligned}
$$

Connaissant la matrice $\mathbf{V}_{11}$ dans la population totale, il est possible d'estimer $\hat{\mathbf{V}}_{12}$ et $\hat{V}_{22}$ à 
partir des variances et covariances observées $\left(\mathbf{V}^{\star}\right)$ dans l'échantillon sélectionné (Lawley, 19431944) :

$$
\begin{aligned}
\hat{\mathbf{v}} & =\left[\begin{array}{ll}
\mathbf{V}_{11} & \hat{\mathbf{V}}_{12} \\
\operatorname{sym} & \hat{\mathbf{V}}_{22}
\end{array}\right] \\
& =\left[\begin{array}{cc}
\mathbf{V}_{11} & \mathbf{V}_{11} \mathbf{V}_{11}^{-1 \star} \mathbf{V}_{12}{ }^{\star} \\
\operatorname{sym} & \mathbf{V}_{22}{ }^{\star}-\mathbf{V}_{21}{ }^{\star}\left(\mathbf{V}_{11}^{-1 \star}-\mathbf{V}_{11}^{-1}{ }^{-1} \mathbf{V}_{11} \mathbf{V}_{11}^{-1}\right) \mathbf{V}_{12}{ }^{\star}
\end{array}\right]
\end{aligned}
$$

Dans le cas d'une sélection divergente par troncature sur une seule variable $\left(x_{1}\right)$, sa variance observée dans l'échantilion sélectionné est augmentée :

$$
v_{11}^{*}=(1+k) v_{11}
$$

où k est un paramètre positif égal à : $\mathrm{k}=t \phi(t) / p, t$ étant le point de troncature sur la distribution de $x_{1}$ $\left(x_{1}<-t\right.$ ou $\left.t<x_{1}\right), \phi(t)$ son ordonnée selon la fonction de densité de la loi de $x_{1}$ et $p$ la proportion d'individus sélectionnés à chaque extrémité.

A partir de $\mathrm{k}$ et des corrélations observées $\left(\mathbf{R}^{\star}\right)$ entre les variables $x_{1}, x_{2}$ et $x_{3}$ dans l'échantillon sélectionné, les corrélations $(\hat{\mathbf{R}})$ dans la population totale s'estiment de la façon suivante :

$$
1 \frac{r_{12}{ }^{*}}{\sqrt{1+k-k r_{12}^{2}}} \quad \frac{r_{13^{*}}}{\sqrt{1+k-k r_{13}^{2}{ }^{*}}}
$$

$$
\hat{\mathbf{R}}=\operatorname{sym}_{\operatorname{sym}} \operatorname{sym}^{\sqrt{1+k-k r_{12}^{2}}} \sqrt{1+k-k r_{13}^{2}{ }^{*}}
$$

La variance d'échantillonnage de l'estimation du coefficient de corrélation entre $x_{1}$ et $x_{2}\left(r_{12}\right)$ à partir des taureaux sélectionnés, $V\left(\widehat{r_{12}}\right)$, peut être calculée en fonction de celle qui existe dans un échantillon de même taille $(n)$ mais dont les taureaux ont été choisis au hasard, $V\left(r_{12}\right)$ :

$$
V\left(\widehat{r_{12}}\right) \cong\left|\frac{\delta r_{12}}{\delta r_{12}}\right|^{2} V\left(r_{12}{ }^{*}\right)=\frac{1+k r_{12}^{2}}{1+k} \cdot V\left(r_{12}\right)
$$

si la variance approchée d'une corrélation est :

$$
V(r) \cong \frac{\left(1-r^{2}\right)^{2}}{n}
$$

\section{RÉSULTATS}

\section{Analyse du dispositif expérimental}

L'échantillonnage des 140 taureaux expérimentaux est représenté sur la figure 1. Sur l'ensemble des 510 taureaux contrôlés, l'indice de sélection $\left(I_{S}\right)$ a une moyenne de $m=100,0$ et un écart type de $\sigma=6,8$ (tableau I). Dans l'échantillon expérimental, la variabilité de cet indice est nettement augmentée, avec un écart type de $\sigma^{*}=10,9$, du fait de l'existence de 2 sous-populations ayant pour valeur moyenne $I_{S}=90,9$ pour les taureaux inférieurs et $I_{S}=110,8$ pour les taureaux supérieurs. Cette augmentation peut être caractérisée par le rapport de la variance observée dans l'échantillon expérimental sur celle observée dans la population totale :

$$
v_{11}{ }^{*} / v_{11}=1+k=(10,9 / 6,8)^{2}=2,58
$$

D'après (1) le dispositif permet, à même effectif contrôlé, de réduire la variance d'échantillonnage des estimées des corrélations entre l'indice de sélection et les autres variables dans la proportion :

$$
V\left(\widehat{r_{12}}\right) / V\left(r_{12}\right)=\left(1+k r_{12}^{2}\right) / 1+k .
$$

Pour des valeurs de $r_{12}$ comprises entre 0 et 0,30 , la variance d'échantillonnage est réduite dans les proportions suivantes:

$$
\begin{array}{lcccc}
r_{12}= & 0,0 & 0,1 & 0,2 & 0,3 \\
V\left(\widehat{r_{12}}\right) / V\left(r_{12}\right)= & 0,39 & 0,39 & 0,41 & 0,44
\end{array}
$$

Ainsi, avec seulement 140 taureaux contrôlés, les corrélations supérieures à 0,05 peuvent être considérées comme significatives $(p<0,05)$ et celles supérieures à 0,14 comme très significatives $(p<0,01)$.

Afin de vérifier la fiabilité du dispositif, les estimations des corrélations obtenues $(\hat{\mathbf{R}})$ à partir de l'échantillon expérimental sont comparées aux valeurs observées dans la popu- 


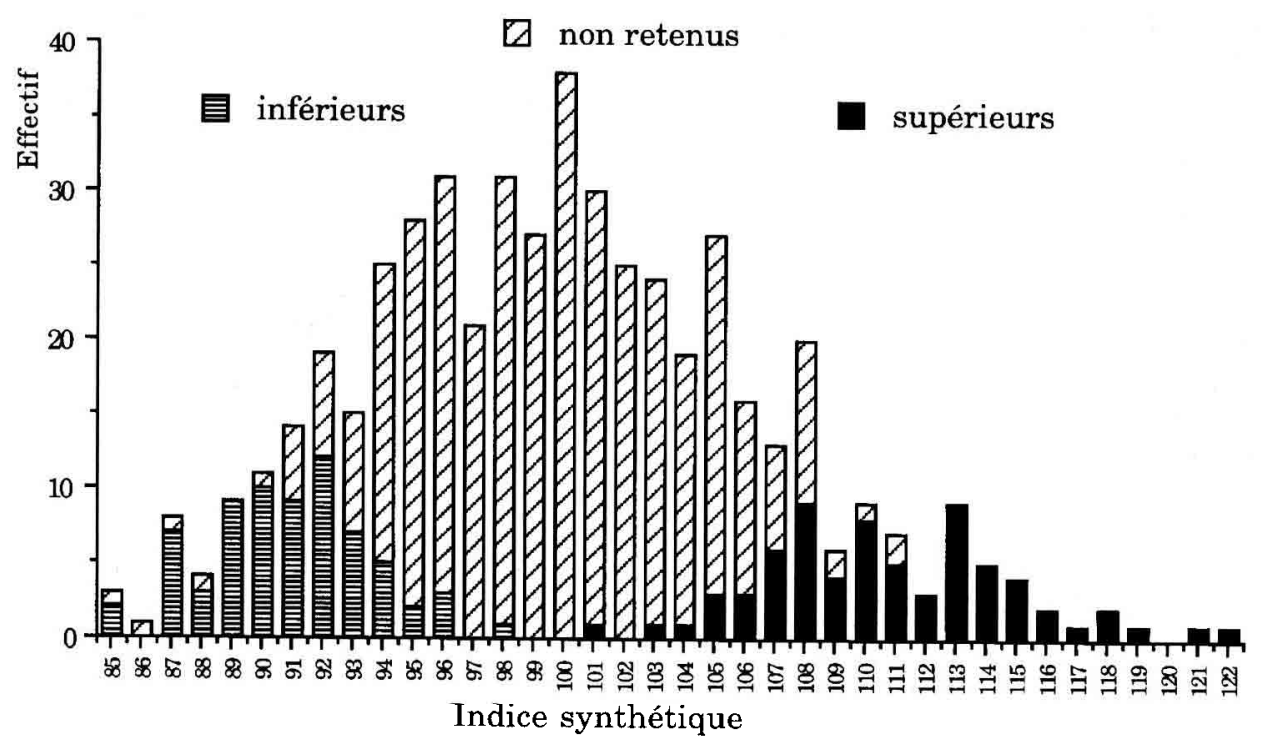

Fig 1. Répartition des taureaux en fonction de leur indice synthétique $\left(I_{S}\right)$.

lation totale (R) pour les cinq indices (tableau II). Malgré des variabilités et des corrélations observées $\left(\mathbf{R}^{\star}\right)$ dans l'échantillon expérimental nettement plus élevées, les paramètres estimés $(\hat{\mathbf{R}})$ sont très proches des paramètres observés dans la population totale $(\mathbf{R})$. Les écarts types estimés ne s'écartent que de $3 \%$ en moyenne des valeurs observées. Les coefficients de corrélation estimés ne s'écartent que de 0,03 en moyenne $(0,11$ au maximum) des coefficients de corrélation observés.

\section{Effets de l'échantillonnage}

Les moyennes des performances enregistrées pendant les contrôles et des critères de composition corporelle sont rapportées dans le tableau III pour chacun des deux échantillons. Le poids vif à la fin des contrôles des 70 taureaux sélectionnés positivement est supérieur de $+96 \mathrm{~kg}(+14 \%)$ à celui des 70 taureaux sélectionnés négativement. Les deux tiers de cette supériorité $(+65 \mathrm{~kg})$ ont été acquis pendant les 18 semaines de contrôle grâce à un gain moyen quotidien nettement supérieur : $+557 \mathrm{~g} /$ jour $(+47 \%)$. Ces mêmes taureaux sélectionnés positivement présentent également une moindre consommation résiduelle : $-1,7 \mathrm{~kg} /$ jour $(-15 \%)$, malgré une consommation journalière supérieure : $+1,0 \mathrm{~kg} /$ jour $(+9 \%)$. Toutes ces différences sont hautement significatives $(p<0,001)$.

La masse des dépôts adipeux totaux (DA) est significativement $(p<0,05)$ plus importante chez les taureaux supérieurs : $+6,5 \mathrm{~kg}(+7 \%)$. En revanche lorsque ces dépôts adipeux sont exprimés en pourcentage du poids vif final, leur teneur (\%DA) est significativement $(p<0,05)$ plus faible : $-0,8 \mathrm{~g} / \mathrm{kg}(-6 \%)$. Si une tendance est observée dans le même sens pour le diamètre des adipocytes : $-1 \mu(-1 \%)$, cette différence n'est toutefois pas significative. 
Tableau I. Moyennes $(\mathrm{m})$ et écarts types $(\sigma)$ des indices dans la population totale et dans l'échantillon expérimental des taureaux retenus.

Taureaux

$\begin{array}{lll}\text { Totaux } & n=510 \\ \text { Retenus } & n=140 \\ \text { Supérieurs } & n=70 \\ \text { Inférieurs } & n=70 \\ & - & -\end{array}$

Les estimées des corrélations $(\hat{\mathbf{R}})$ entre les indices et les critères de composition cor-

$90,9 \quad 2,5$
Indices

Poids final
$m \quad \sigma$

Consom résid

$m$

$\sigma$

$\begin{array}{rr}100,0 & 8,7 \\ 100,6 & 12,7 \\ 111,7 & 5,0 \\ 89,4 & 5,0\end{array}$

100,0

6,0

$100,9 \quad 9,2$

$108,9 \quad 4,5$

porelle sont rapportées dans le tableau IV. Les corrélations sont relativement faibles. Or, les critères de composition corporelle ont été estimés par une technique indirecte qui présente une certaine imprécision :

Tableau II. Écarts types et coefficients de corrélation entre les indices.

Écarts types dans la population totale

Observés $(n=510)$

Estimés à partir de l'échantillon is

6,8

6,8
$\mathrm{I}_{\mathrm{CR}}$

$\mathrm{I}_{\mathrm{DM}}$

Ios

Corrélation entre indices a

$$
\begin{aligned}
& I_{S}=\text { indice synthétique } \\
& l_{P F}=\text { indice poids final } \\
& I_{C R}=\text { indice consommation résiduelle } \\
& I_{D M}=\text { indice développement musculaire } \\
& +0,53+0,60 \\
& -0,42 \\
& +0,88 \\
& -0,90 \\
& +0,38 \quad+0,37 \\
& +0,88 \\
& -0,91 \\
& +0,36 \\
& +0,39 \\
& +0,95 \\
& -0,58 \\
& +0,46 \quad+0,47 \\
& -0,60 \\
& +0,48 \quad+0,48 \\
& \begin{array}{llll}
-0,96 & -0,83 & -0,23 & -0,20 \\
-0,18 & -0,23
\end{array} \\
& +0,57 \quad+0,62 \quad-0,47 \quad+0,34 \\
& I_{D S}=\text { indice développement squelettique }
\end{aligned}
$$

\footnotetext{
a Au dessus de la diagonale : 1 re ligne : corrélations observées $(\boldsymbol{R})$ dans la population totale $(n=510) ; 2$ ligne : corrélations estimées $(\hat{\mathbf{R}})$ à partir de l'échantillon expérimental ; en dessous de la diagonale : corrélations observées $\left(R^{\star}\right)$ dans l'échantillon expérimental $(n=140)$.
}$$
7,9
$$$$
7,6
$$$$
7,9
$$$$
8,5
$$$$
6,1
$$ 
Tableau III. Effets de l'échantillonnage sur les performances zootechniques moyennes.

\begin{tabular}{|c|c|c|c|c|c|}
\hline & Écart type a & $\begin{array}{c}\text { Coefficient de } \\
\text { variation }\end{array}$ & & reaux & $\begin{array}{c}\text { Rapport } \\
\text { Sup/Inf }\end{array}$ \\
\hline & & & $\begin{array}{c}\text { Inférieurs } \\
(\mathrm{n}=70)\end{array}$ & $\begin{array}{c}\text { Supérieurs } \\
(\mathrm{n}=70)\end{array}$ & \\
\hline- & - & . & - & & - \\
\hline Âge initial (j) & 28 & $7,6 \%$ & 367 & 369 & $1,01 \mathrm{~ns}$ \\
\hline Poids initial $(\mathrm{kg})$ & 47 & $8,7 \%$ & 534 & 565 & $1,06^{\star \star *}$ \\
\hline Poids final $(\mathrm{kg})$ & 54 & $7,4 \%$ & 684 & 780 & $1,14^{\star \star \star}$ \\
\hline GMQ (g/jour) & 231 & $16,3 \%$ & 1181 & 1738 & $1,47^{* * *}$ \\
\hline Consom journalière $(\mathrm{kg} / \mathrm{jour})$ & 0,98 & $8,4 \%$ & 11,23 & 12,25 & $1,09^{* *}$ \\
\hline Consom résiduelle $(\mathrm{kg} / \mathrm{jour})^{\mathrm{b}}$ & 0,69 & $5,9 \%$ & $+0,58$ & $-1,13$ & $0,85^{* * *}$ \\
\hline Dépôts adipeux $(\mathrm{kg})$ & 16,2 & $18,0 \%$ & 86,5 & 93,0 & $1,07^{*}$ \\
\hline \% Dépôts adipeux (\%) & 1,9 & $15,4 \%$ & 12,6 & 11,8 & $0,94^{*}$ \\
\hline Diam adipocytes $(\mu)$ & 13,7 & $16,8 \%$ & 82,0 & 81,0 & $0,99 \mathrm{~ns}$ \\
\hline
\end{tabular}

a Écarts types observés pour les variables de croissance et de consommation, et écarts types estimés pour les variables de composition. b Coefficient de variation et consommation résiduelle rapportés à la consommation journalière moyenne ; ${ }^{*} p<0,05,{ }^{\star * \star} p<0,001$, ns : non significatif.

l'écart type résiduel de l'équation de prédiction de la masse adipeuse par la mesure de l'espace de diffusion de l'eau lourde représente $13 \%$ de la moyenne et celui de la teneur $8 \%$ (Robelin, 1982). De ce fait, cette imprécision ne peut que réduire le degré de liaison entre ces critères d'adiposité (DA et \%DA) et les indices. II existe toutefois une relation positive marquée $(r=+0,30)$, tout à fait prévisible, entre l'indice de poids final $\left(I_{P F}\right)$ et la masse des dépôts adipeux totaux (DA). En revanche, la teneur en dépôts adipeux (\%DA) est indépendante $(r=+0,01)$ de cet indice alors qu'elle présente une corrélation légèrement positive $(r=+0,16)$ avec l'indice de consommation résiduelle $\left(I_{C R}\right)$. La combinaison de ces deux indices en un indice synthétique $\left(I_{S}\right)$ a pour conséquence que ce dernier est corrélé légèrement à la fois positivement $(r=+0,16)$ avec la masse des dépôts adipeux totaux, et négativement $(r=-0,09)$ avec la teneur en dépôts adipeux.

Les corrélations entre le diamètre des adipocytes (Diam) et les indices de poids final $\left(I_{P F}\right)$, de consommation résiduelle $\left(I_{C R}\right)$ et synthétique $\left(I_{S}\right)$ sont intermédiaires par rapport aux corrélations qui existent entre ces trois indices d'une part et la masse (DA) ou la teneur (\%DA) en dépôts adipeux d'autre part.

\section{DISCUSSION}

\section{Variabilité de la croissance, de l'efficacité alimentaire et de la composition corporelle}

Les valeurs élevées des coefficients de variation de la masse et de la teneur en dépôts adipeux (CV $=18$ et $15 \%$ respectivement), doubles de celle du poids final ( $\mathrm{CV}=7 \%$ ), et l'absence de relation entre l'indice poids final $\left(I_{P F}\right)$ et la teneur en dépôts adipeux (\%DA) montrent qu'il existe une grande variabilité individuelle de la composition corporelle, indépendante de la vitesse de croissance chez ces animaux de 
Tableau IV. Coefficients de corrélations estimés entre les indices et les critères de composition corporelle.

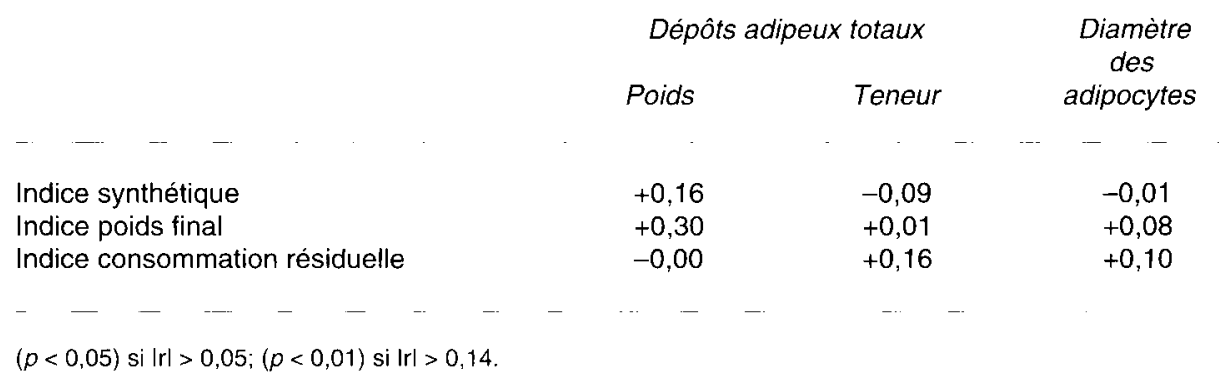

même race, de même sexe, de même âge et contrôlés pendant 6 mois dans une même station avec le même régime alimentaire. Cette variabilité individuelle, ou phénotypique, est la résultante de l'effet des gènes de l'individu et de tous les effets de l'environnement qui ne peuvent être identifiés. Les travaux publiés montrent que, dans des conditions standardisées de contrôle en station, la part de la variabilité individuelle d'origine génétique, ou héritabilité, est d'environ $h^{2}=0,4$ pour les critères de croissance, alors qu'elle s'élève à plus de $h^{2}=0,5$ pour les critères de composition corporelle (Renand et al, 1992). L'amélioration génétique de la croissance musculaire par sélection est donc tout à fait envisageable, et sera d'autant plus efficace, si la sélection peut porter non seulement sur leur croissance en vif, mais inclure également une mesure de leur composition corporelle.

Les corrélations estimées entre l'indice de poids final $\left(I_{P F}\right)$ d'une part et la masse (DA) ou la teneur (\%DA) en dépôts adipeux d'autre part ( $r=+0,30$ et $r=+0,01$ respectivement) sont plus faibles que les moyennes qui peuvent être calculées à partir des quelques corrélations publiées avec de jeunes bovins contrôlés en station à âge constant : $r=+0,45$ et $r=+0,16$ respecit: vement (Kräusslich et al, 1970; Koch et al, 1982; Weiher, 1982). Or, comme pour toutes corrélations entre deux caractères, ces corrélations inter individus ( $r$ ), ou corrélations phénotypiques, entre poids et adiposité, résultent d'une corrélation entre les effets génétiques $(\mathrm{rg})$ d'une part et d'une corrélation due aux effets du milieu $\left(r_{e}\right)$ d'autre part, selon la formule classique:

$$
r=r_{g} h_{1} h_{2}+r_{e} \sqrt{ } 1-h_{1}^{2} \sqrt{ } 1-h_{2}^{2}
$$

où $h_{1}^{2}$ et $h_{2}^{2}$ sont les coefficients d'héritabilité.

Ces effets du milieu interviennent essentiellement par l'intermédiaire du niveau alimentaire de chaque individu. II est bien connu que toute augmentation de ce niveau alimentaire induit une augmentation concomitante de la vitesse de croissance et de la part des dépôts adipeux dans le croît (Geay et al, 1976 ; Robelin et Geay, 1984). Ceci est confirmé par les corrélations dues aux effets du milieu $\left(r_{e}\right)$ qui sont nettement positives dans les trois travaux cités précédemment: $r_{e}=+0,55$ entre le poids final et le poids de gras et $r_{e}=+0,36$ entre le poids final et la teneur en gras des carcasses.

Dans ces trois travaux, les corrélations génétiques entre le poids final d'une part et le poids ou la teneur en gras d'autre part étaient respectivement de $r_{g}=+0,33$ et $r_{g}=+0,01$. Ces corrélations génétiques $\left(r_{g}\right)$ sont nettement moins marquées que celles dues aux effets du milieu $\left(r_{e}\right)$. Les corréla- 
tions phénotypiques ( $r$ ) sont intermédiaires. Dans la population de la présente étude, bien qu'il soit impossible d'estimer la part due aux effets génétiques et celle due aux effets du milieu, les faibles valeurs des corrélations phénotypiques $(r)$ entre l'indice de poids final et les critères d'adiposité indiquent que les effets dus au milieu n'ont certainement qu'une influence réduite sur la variabilité et les corrélations.

Comparativement aux corrélations estimées pour l'indice de poids final $\left(l_{P F}\right)$, les corrélations entre l'indice de consommation résiduelle $\left(I_{C R}\right)$ et l'adiposité sont plus favorables : absence de relation avec la masse et corrélation légèrement positive $(r=+0,16)$ avec la teneur en dépôts adipeux totaux. C'est dire que les animaux qui ont une meilleure efficacité alimentaire ont tendance à déposer proportionnellement moins de gras. Ainsi une sélection basée sur la mesure de la consommation résiduelle aurait tendance à réduire l'adiposité alors qu'une sélection sur le seul poids final ne permettrait pas de modifier la proportion de muscles et de gras déposés.

Bien que dans la littérature il n'y ait pas d'études avec des mesures similaires, quatre travaux permettent de comparer les corrélations qui existent entre l'adiposité d'une part et la vitesse de croissance ou l'indice de consommation d'autre part (Dickerson et al, 1974 ; Mavrogenis et al, 1978 ; Brelin et Brännäng, 1982 ; McNeil et al, 1991). Dans ces travaux, les corrélations phénotypiques moyennes entre le gain moyen quotidien ou l'indice de consommation d'une part et les critères d'adiposité (poids des dépôts adipeux ou épaisseur du gras sous cutané de la carcasse) d'autre part sont respectivement de $r=+0,32$ et $r=+0,09$, alors que les corrélations génétiques sont respectivement de $r_{g}=+0,19$ et $r_{g}=+0,04$ et les corrélations dues au milieu de $r_{e}=0,46$ et $r_{e}=+0,17$, en moyenne.

Dans la présente étude, la corrélation entre l'indice de consommation résiduelle
$\left(l_{C R}\right)$ et la teneur en dépôts adipeux (\%DA) due aux effets du milieu est certainement plus faible que la valeur moyenne observée dans ces quatre travaux $\left(r_{e}=+0,17\right)$. En effet Geay et Robelin (1979) ont montré qu'il existe un niveau alimentaire optimal correspondant à une efficacité maximale et que toute augmentation de l'apport énergétique au-delà de ce niveau conduit à un fort accroissement de l'adiposité et induit une relation positive entre l'indice de consommation et l'adiposité. C'est le cas des travaux précédemment cités, réalisés avec des régimes assez énergétiques et des races précoces, où la corrélation due au milieu est positive $\left(r_{e}=+0,17\right)$. Dans la présente étude, menée avec des animaux de race à viande tardive, les quantités d'aliments distribués visent à atteindre cet optimum et à limiter ainsi l'incidence des variations de niveau alimentaire non liées à l'animal sur la relation entre efficacité alimentaire et adiposité.

Si la prise en compte de l'efficacité alimentaire est certainement favorable à la sélection des individus qui déposent proportionnellement plus de muscles que de gras, il est évident que cette sélection indirecte ne peut être qu'imparfaite. En effet l'efficacité alimentaire est un caractère complexe qui fait intervenir la capacité d'ingestion, la capacité à digérer et à métaboliser l'énergie brute contenue dans les aliments, les besoins d'entretien, les rendements énergétiques de la fixation des protéines et des lipides, ainsi que les quantités relatives de protéines et de lipides fixées. Parmi toutes ces composantes, il existe une forte variabilité génétique de la composition corporelle, aussi bien intrarace qu'entre races (Cundiff et al, 1986 ; Renand et al, 1992). Si des variations génétiques n'ont pu être mises en évidence pour la capacité digestive ou les rendements énergétiques (Warwick et Cobb, 1975), il existe en revanche des résultats montrant des différences de besoins d'entretien entre races 
(Vermorel et al, 1976 ; Webster et al, 1983 ; Ferrell et Jenkins, 1985) et quelques résultats sur l'existence d'une variabilité génétique intrarace (Andersen, 1980 ; Taylor et al, 1981 ; Carstens et al, 1989 ; Hotovy et al, 1991). La relation génétique entre ces besoins d'entretien et la composition corporelle est également complexe et contribue à affaiblir la relation entre cette dernière et l'efficacité alimentaire. Si la moindre activité métabolique dans les dépôts adipeux est un élément favorable pour des besoins d'entretien plus faibles chez les animaux gras par rapport aux animaux maigres (Geay, 1984 ; Webster, 1985), il apparaît clairement qu'une grande part de l'énergie non utilisée pour la croissance est utilisée dans l'intense activité métabolique des organes internes et que le développement relatif de ces derniers a une incidence importante sur l'efficacité alimentaire (Ferrell et Jenkins, 1985 ; Jenkins et al, 1986 ; Webster, 1989). Ortigues et al (1995) ont montré que les activités métaboliques du tractus digestif et du foie sont trois à quatre et six à 13 fois plus élevées que celles du membre postérieur. Ainsi, le plus grand développement du tractus digestif des races laitières est un élément d'explication des besoins d'entretien supérieurs dans ces races comparativement aux races à viande britanniques (Truscott et al, 1983 ; Webster et al, 1983 ; Taylor et al, 1986) ou aux races à viande continentales (Vermorel et al, 1976).

Quoi qu'il en soit et malgré les faibles relations estimées dans la présente étude entre la croissance ou l'efficacité alimentaire d'une part et l'adiposité d'autre part, l'échantillonnage sur l'indice synthétique $\left(I_{S}\right)$ a tout de même permis de retenir des taureaux qui avaient non seulement des différences importantes de poids à la fin des contrôles $(+96 \mathrm{~kg}$, soit $+14 \%)$ et de consommation résiduelle $(-1,7 \mathrm{~kg} / \mathrm{jour}$, soit $-15 \%$ ), mais dont la teneur en dépôts adipeux était également significativement plus faible $(-8 \mathrm{~g} / \mathrm{kg}$, soit $-6 \%)$. L'échantillon- nage sur cet indice synthétique a très certainement permis une discrimination des taureaux sur leur croissance musculaire.

L'efficacité d'une sélection basée sur cet indice pour améliorer effectivement la croissance musculaire ne peut être appréhendée par les seuls résultats phénotypiques de la présente étude. Elle est actuellement en cours de vérification par le contrôle sur descendance de 60 taureaux parmi les 140 de l'échantillon : 30 parmi les 70 taureaux supérieurs et 30 parmi les 70 taureaux inférieurs. Les résultats préliminaires obtenus sur 317 taurillons montrent qu'à 17 mois les carcasses des descendants des taureaux supérieurs sont plus lourdes $(+13 \mathrm{~kg}$, soit $+13 \%$ ) et contiennent $6 \mathrm{~g} / \mathrm{kg}$ de muscles en plus et $8 \mathrm{~g} / \mathrm{kg}$ de gras en moins que celles des descendants des taureaux inférieurs (Renand et al, 1994).

\section{Estimation de la composition corporelle par la mesure du diamètre des adipocytes}

Pour améliorer l'efficacité de la sélection sur la croissance musculaire dans ces stations de contrôle individuel, il est possible d'intégrer comme critère supplémentaire la mesure du diamètre moyen des adipocytes du tissu adipeux sous-cutané, prélevés par biopsie. Les corrélations entre ce diamètre (Diam) d'une part et la masse (DA) ou la teneur $(\% \mathrm{DA})$ en dépôts adipeux sont de $r=$ $+0,53$ et $r=+0,56$ respectivement. Bien que relativement élevées, ces corrélations restent limitées par le fait que les dépôts adipeux sont estimés et non pas mesurés. Comme déjà signalée à propos des relations avec les indices, l'imprécision de cette estimation ne peut que réduire le degré de liaison entre la mesure du diamètre des adipocytes (Diam) et les critères d'adiposité (DA et \%DA).

Ces corrélations ne peuvent être parfaites car les mesures effectuées ne repré- 
sentent pas tout à fait la même chose en termes biologiques. L'estimation des dépôts adipeux totaux est indirectement basée sur l'estimation des lipides corporels totaux à travers la mesure de l'espace de diffusion d'un marqueur. II existe en effet une étroite relation entre la teneur en eau et en lipides du fait de la stabilité de la composition de la masse maigre, ainsi qu'une étroite relation entre dépôts adipeux totaux et lipides corporels totaux chez les bovins en croissance (Robelin et Geay, 1978). La mesure du diamètre des adipocytes traduit l'activité de synthèse et de stockage dans un dépôt adipeux particulier, le gras de couverture. De plus, Robelin (1981) a montré que, chez les jeunes bovins en croissance, $70 \%$ de l'accroissement des lipides corporels totaux sont liés à l'hypertrophie des adipocytes.

L'intégration de cette mesure dans un indice de sélection nécessitera la connaissance des paramètres génétiques en relation avec les autres caractères sélectionnés. II est déjà possible de constater que les corrélations entre ce diamètre d'une part et la masse ou la teneur en dépôts adipeux sont identiques : $r=+0,53$ et $r=+0,56$ respectivement. De plus l'analyse des corrélations de ce diamètre (Diam) avec l'indice de poids final $\left(I_{P F}\right)$ ou avec l'indice de consommation résiduelle $\left(l_{C R}\right)$, comparativement à celles qui existent entre ces deux indices et la masse (DA) ou la teneur (\%DA), montre que cette mesure de la taille des adipocytes se comporte comme un caractère intermédiaire entre masse et teneur. Si la masse musculaire d'un taureau peut être considérée égale à $0,55 \mathrm{PF}$ - DA, la précision de son estimation, en termes phénotypiques, peut être augmentée de $5 \%$ par l'utilisation de la mesure de la taille des adipocytes en sus des indices $\left(I_{P F}\right.$ et $I_{C R}$ ), la part de la variance expliquée passant de $\mathrm{R}^{2}=81 \%$ à $\mathrm{R}^{2}=86 \%$.

Les valeurs des coefficients de corrélation entre ce diamètre et les dépôts adipeux totaux sont à rapprocher de la corrélation phénotypique moyenne $(r=+0,64)$ observée entre l'épaisseur du gras de couverture d'une part et le poids ou la teneur en gras des carcasses d'autre part dans les trois travaux suivants (Mason et al, 1972 ; Dickerson et al, 1974 ; Koch et al, 1982). Dans ces travaux, il apparaissait que les corrélations génétiques étaient très proches des corrélations phénotypiques observées, avec une valeur moyenne de $r_{g}=+0,67$. II est donc possible d'espérer que, chez les taureaux de la présente étude, la corrélation génétique entre le diamètre des adipocytes et la teneur en dépôts adipeux soit du même ordre de grandeur que la corrélation phénotypique, c'est-à-dire proche de $+0,6$. Ces paramètres sont en cours d'estimation grâce au contrôle sur descendance d'une partie de ces 140 taureaux.

\section{CONCLUSION}

Les résultats de cette étude montrent qu'en l'absence de prédicteur efficace de la composition corporelle la sélection simultanée des taureaux sur leur poids final et leur consommation résiduelle est appropriée pour sélectionner la croissance musculaire alors qu'une sélection sur la seule croissance en vif ne permet pas de modifier favorablement la composition corporelle. Ces résultats phénotypiques sur les taureaux en contrôle individuel doivent être confirmés au niveau génétique par la mesure de la croissance musculaire et la composition corporelle de leurs descendants. Indépendamment de l'intensité de sa relation avec la composition corporelle, la consommation résiduelle représente un des principaux objectifs d'amélioration de la production de viande bovine en complément de la croissance musculaire.

Une sélection encore plus efficace des taureaux pour améliorer la croissance musculaire est possible en intégrant la mesure de la taille des adipocytes aux deux critères 
précédents. La pondération de ces trois critères pour un objectif de sélection sur l'efficacité de la production de viande bovine nécessite de connaître les paramètres génétiques de ces critères et non plus seulement les variabilités et relations phénotypiques mises en évidence dans la présente étude. Cette mesure de la taille des adipocytes requiert toutefois quelques manipulations en laboratoire qui peuvent apparaître comme un facteur limitant à sa mise en $œ u v r e$ en routine dans les stations de contrôle individuel.

\section{REMERCIEMENTS}

Les centres de production de semence UCATRC et OGER sont remerciés pour avoir mis leurs taureaux à disposition pour cette expérimentation. Cette étude a été réalisée dans le cadre de l'AIP "Tissus adipeux» de l'Inra.

\section{RÉFÉRENCES}

Andersen BB (1980) Feeding trials describing net requirements for maintenance as dependent on weight, feeding level, sex and genotype. Ann Zootech 29, 85-92

Andersen BB, De Baerdemaeker A, Bittante G, Bonaïti $B$, Colleau JJ, Fimland $E$, Jansen J, Lewis WHE, Politiek RD, Seeland G, Teehan TJ, Wermeister F (1981) Performance testing of bulls in Al: report of a working group of the commission on cattle production. Livest Prod Sci 8, 101-119

Brelin B, Brännäng E (1982) Phenotypic and genetic variation in feed efficiency of growing cattle and their relationship with growth rate, carcass traits and metabolic efficiency. Swed J Agric Res 12, 29-34

Carstens GE, Johnson DE, Johnson KA, Hotovy SK, Szymanski TJ (1989) Genetic variation in energy expenditure of monozygous twin beef cattle at 9 and 20 months of age. In : Energy metabolism of farm animals. Eur Assoc Anim Prod 43, 312-315

Cundiff LV, Gregory KE, Koch RM, Dickerson GE (1986) Genetic diversity among cattle breeds and its use to increase beef production efficiency in a temperate environment. In : 3rd World Congress Genet Applied Livest Prod, Lincoln, NE, vol IX, 271-282

Dickerson GE, Künzi N, Cundiff LV, Koch RM, Arthaud $\mathrm{VH}$, Gregory KE (1974) Selection criteria for efficient beef production. J Anim Sci 39, 659-673
Ferrell CL, Jenkins TG (1985) Energy utilization by Hereford and Simmental males and females. Anim Prod 41, 53-61

Geay $Y$ (1984) Energy and protein utilization in growing cattle. J Anim Sci 58, 766-778

Geay $Y$, Robelin J (1979) Variation of meat production capacity in cattle due to genotype and level of feeding: genotype-nutrition interaction. Livest Prod SCi 6, 263-276

Geay $Y$, Micol D (1988) Alimentation des bovins en croissance et à l'engrais. In : Alimentation des bovins, ovins et caprins ( $R$ Jarrige, ed), Inra, Paris, 213-247

Geay $Y$, Beranger C, Jarrige R (1971) Variations de la quantité d'énergie ingérée par des taurillons à l'engrais. In : $10^{\circ}$ Congrès international de zootechnie, Paris, France

Geay Y, Robelin J, Béranger C (1976) Influence du niveau alimentaire sur le gain de poids vif et la composition de la carcasse de taurillons de différentes races. Ann Zootech 25, 287-298

Hotovy SK, Johnson KA, Johnson DE, Carstens GE, Bourdon RM, Seidel GE (1991) Variations among twin beef cattle in maintenance energy requirements. $J$ Anim Sci 69, 940-946

Jenkins TG, Ferrell CL, Cundiff LV (1986) Relationship of components of the body among mature cows as related to size, lactation potential and possible effects on productivity. Anim Prod 43, 245-254

Koch RM, Cundiff LV, Gregory KE (1982) Heritabilities and genetic, environmental and phenotypic correlations of carcass traits in a population of diverse biological types and their implication in selection programs. J Anim Sci 55, 1319-1329

Kräusslich H, Averdunk G, Gottschalk A, Schmitter W, Schuman H, Schwarz E (1970) Die Besamungszucht beim Rind in Bayern. Bayer Landw Jahrbuch 47, 3-85

Lawley DN (1943-44) A note on Karl Pearson's selection formulae. Proc Roy Soc Edin, Sect A 62, 28-30

McNeil MD, Bailey DRC, Urick JJ, Gilbert RP, Reynolds WL (1991) Heritabilities and genetic correlations for postweaning growth and feed intake of beef bulls and steers. J Anim Sci 69, 3183-3189

Mason IL, Vial VE, Thompson R (1972) Genetic parameters of beef characters and the genetic relationship between meat and milk production in British Friesian cattle. Anim Prod 14, 135-148

Mavrogenis AP, Dillard EU, Robison OW (1978) Genetic analysis of postweaning performance of Hereford bulls. J Anim Sci 47, 1004-1013

Ménissier $F$ (1988) La sélection des races bovines à viande spécialisées en France. In : 3rd World Congr on sheep and beef cattle breeding, Paris, France, vol II, 215-236

Ménissier F, Renand G, Colleau JJ, Gaillard $J$ (1986) Amélioration génétique de la production de viande 
bovine : orientations, objectifs et méthodes de sélection des aptitudes bouchères. In : Production de viande bovine (D Micol, ed), Inra, Paris, 101-146

Ortigues I, Martin C, Durand D, Vermorel M (1995) Circadian changes in energy expenditure in the preruminant calf: whole animal and tissue level. J Anim Sci $73,552-564$

Pearson K (1903) Mathematical contribution to the theory of evolution. XI. On the influence of natural selection on the variability and correlation of organs. Phil Trans Roy Soc Lond, Sect A 200, 1-66

Renand G, Plasse D, Andersen BB (1992) Genetic improvement of cattle for growth and carcass traits. In : Beef cattle production ( $\mathrm{A}$ Jarrige, $\mathrm{C}$ Béranger, eds). Elsevier, Amsterdam, vol C5, 87-108

Renand G, Berge P, Picard B, Robelin J, Geay Y, Krauss $D$, Ménissier F (1994) Genetic parameters of beef production and meat quality traits of young Charolais bulls progeny of divergently selected sires. In : 5th World Congress Genet Applied Livest Prod, Guelph, ON, Canada, vol 19, 446-449

Robelin J (1981) Cellularity of bovine adipose tissues: developmental changes from 15 to 65 percent mature weight. J Lipid Res 22, 452-457

Robelin J (1982) Estimation of body composition by dilution techniques in nutrition experiments. Beretn fra Stat Husdyr forsog 524, 107-117

Robelin J, Geay Y (1978) Estimation de la composition chimique du corps entier des bovins à partir du poids des dépôts adipeux totaux. Ann Zootech 27, 159167

Robelin J, Daenicke R (1980) Variations of net requirements for cattle growth with liveweight, liveweight gain, breed and sex. Ann Zootech 29, 99-118

Robelin J, Geay Y (1984) Body composition as affected by physiological status, breed, sex and diet. In : Herbivore nutrition in sub-tropics (FMC Gilchrist, RI Mackie, eds), The Science Press, Craighall, 525-548
Robelin J, Agabriel J (1986) Estimation de l'état d'engraissement des bovins vivants à partir de la taille des cellules adipeuses. Bull Tech CRZV Theix, INRA 66, 37-41

Taylor SCS, Turner HG, Young GB (1981) Genetic control of equilibrium maintenance efficiency in cattle. Anim Prod 33, 179-194

Taylor SCS, Thiessen RB, Murray J (1986) Inter-breed relationship of maintenance efficiency to milk yield in cattle. Anim Prod 43, 37-61

Truscott TG, Wood JD, Gregory NG, Hart IC (1983) Fat deposition in Hereford and Friesian steers. 3. Growth efficiency and fat mobilization. J Agric Sci 100, 277284

Vermorel M, Bouvier JC, Geay Y (1976) The effect of genetic type (normal and double-muscled Charolais and Friesian) on energy utilization by growing cattle at 2 and 16 months of age. In : Energy metabolism of farm animals, Eur Assoc Anim Prod 19, 217-220

Warwick EJ, Cobb EH (1975) Genetic variation in nutrition of cattle for meat production. In : The effect of genetic variance on nutritional requirements of animals, Proc Symp Nation Academy Sci, Univ Maryland, MD, États-Unis, 3-18

Webster AJF (1985) Differences in the energetic efficiency of animal growth. J Anim Sci61, 92-103

Webster AJF (1989) Bioenergetics, bioengineering and growth. Anim Prod 48, 249-269

Webster AJF, Smith JS, Mollison GS (1983) Energy requirements of growing cattle: effects of sire breed, plane of nutrition and season on predicted basal metabolism. In : Energy metabolism of farm animals, Eur Assoc Anim Prod 29, 84-95

Weiher $O$ (1982) Effektive Gestaltung der Leistungsprüfung von Fleischrindbullen bei besonderer Berücksichtigung der Zuchtwertschätzung unter Feldbedingungen. Thèse doct sci agric, Wilhem-Pieck Univ, Rostock, Allemagne, $207 \mathrm{p}$ 